DOI: $10.12731 / 2306-1561-2013-4-27$

\title{
EFFECTIVE PRICE POLICY IN THE CENTRALIZED SUPPLY CHAIN SPARE PARTS
}

\section{Vasiliev D.A.}

\section{Abstract}

The paper considers the problem of management of logistics based on a centralized scheme. The problem is the definition of agreed prices, which on the one hand, provide maximum income an organization engaged in the supply side (center), and on the other make more profitable for consumer organizations use a centralized scheme. In the game setting, consumers can change their proposed price. This creates the situation of the game. When profitability of centralized procurement scheme consumers competes for inclusion in the centralized scheme that encourages them to reduce the prices offered.

Keywords: pricing, supply chain, spare parts, performance and supply.

\section{УДК 33}

\section{ЭФФЕКТИВНОСТЬ ЦЕНОВОЙ ПОЛИТИКИ В ЦЕНТРАЛИЗОВАННОЙ СХЕМЕ ПОСТАВОК ЗАПАСНЫХ ЧАСТЕЙ}

\section{Васильев Д.А.}

\section{Аннотация}

В статье рассматривается задача управления материально-техническим снабжением на основе централизованной схемы. Задача связана с определением согласованных иен, которые, с одной стороны, обеспечивают максимум дохода организации, осуществляющей снабжение (иентра), а с другой - делают более выгодным для организаций-потребителей использование централизованной схемы. В игровой постановке потребители могут менять предлагаемые ими ичены. Это и создает игровую ситуаџию. При выгодности централизованной схемы снабжения потребители конкурируют за право включения в централизованную схему, что побуждает их снижать предлагаемые иены.

Ключевые слова: ценовая политика, схема поставок, запасные части, эффективность, снабжение.

\section{Описание модели}

В централизованной схеме снабжения вопросы материально-технического обеспечения берет на себя центр, заключающий договор с организациями- 
потребителями [1 - 10]. Центр проводит оптовые закупки продукции у производителей, что позволяет ему покупать по более низким ценам и, за счет этого, обеспечивать привлекательность централизованной схемы для потребителей.

В работе решена задача снабжения одним видом продукции. Пусть в регионе имеется $\mathrm{n}$ организаций - потенциальных потребителей продукции данного вида. Обозначим через $c_{i}$ цену, по которой i-ый потребитель согласен приобретать продукцию у центра, а через $v_{i}$ - количество продукции, требуемое $i$-му потребителю в рассматриваемый период времени. Очевидно, что потребитель $i$ будет выбирать централизованную схему снабжения если цена продукции у центра, которую мы будем обозначать через $q$, будет меньше или равна сі, то есть $q \leq c_{i}$. Таким образом, количество продукции, которое будет заказано центру равно сумме потребностей тех потребителей, для которых централизованная схема является выгодной.

Обозначим через $P(q)$ множество потребителей, выбирающих централизованную схему снабжения при цене продукции центра равной $q$. Тогда количество продукции, заказываемое у центра, можно записать в следующем виде:

$$
V(q)=\sum_{i \in P(q)} v_{i}
$$

Зависимость $V(q)$ имеет вид, показанный на рисунке 1. Это кусочно-постоянная, непрерывная слева, убывающая функция $q$.

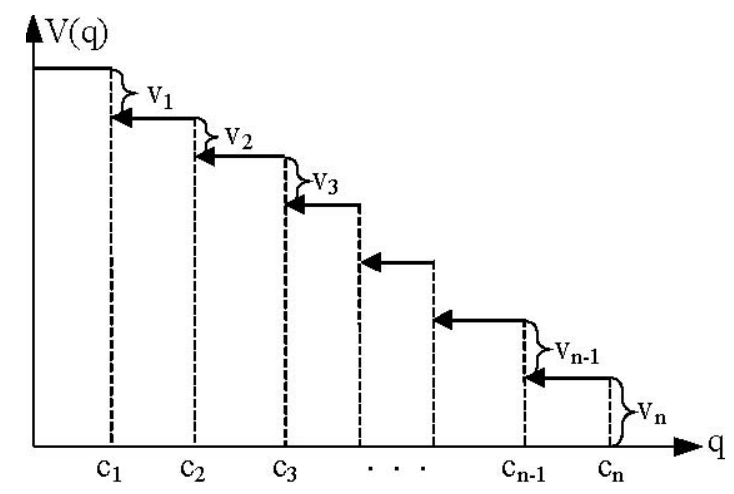

\section{Рисунок 1 - Общее количество заказов центру}

Примем, что центр закупает продукцию у одного производителя, получая скидки к оптовой цене при больших объемах закупок. Обозначим через $b(V)$ цену продукции производителя при объеме закупок $V$. Очевидно, что $b(V)$ также убывающая функция $V$ (как правило, кусочно-постоянная). Прибыль центра при цене продажи потребителям q составит

$$
P=(q-b) * V(q) \text {. }
$$

В данном случае мы полагаем, что транспортные расходы на доставку продукции от производителя центру входят в цену $b(V)$, а транспортные расходы на доставку продукции от центра потребителям производятся за счет потребителей. Задача 
заключается в определении цены $q$, которая обеспечит максимум прибыли центра. Эта цена называется согласованной ценой, поскольку она выгодна и потребителям, и центру.

\section{Метод решения задачи}

Для решения задачи перейдем от функции $V(q)$ (рисунок 1) к обратной функции $q(V)$. Эта функция показывает, какую максимальную цену может установить центр для того, чтобы обеспечить объем заказа $V$. Эта функция также является убывающей, кусочно-постоянной и непрерывной слева (рисунок 2).

Выражение (2) можно записать в виде зависимости прибыли от объема закупок центра:

$$
\Pi(V)=[q(V)-b(V)] * V .
$$

Если обозначить разность цен $[q(V)-b(V)]$ через $\varepsilon(V)$, то выражение (3) примет вид

$$
\Pi(V)=\varepsilon(V) * V
$$

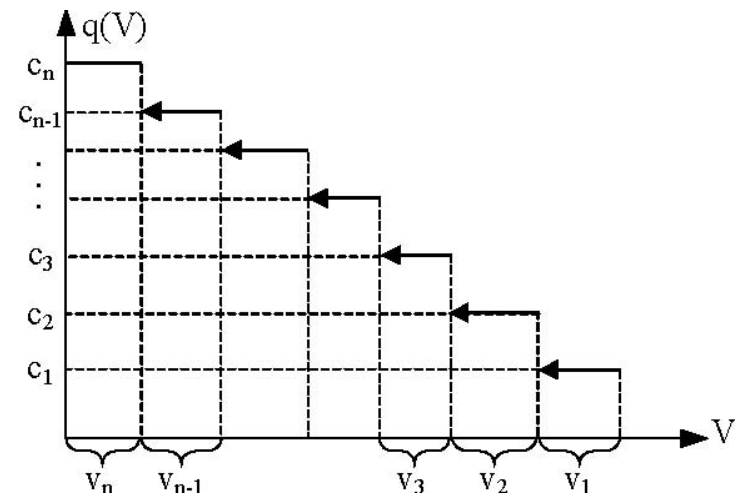

Рисунок 2 - Определение оптовой цены

Геометрически величина $\Pi(V)$ равна площади прямоугольника со сторонами $\varepsilon(V)$ и $V$ (Рисунок 3$)$.

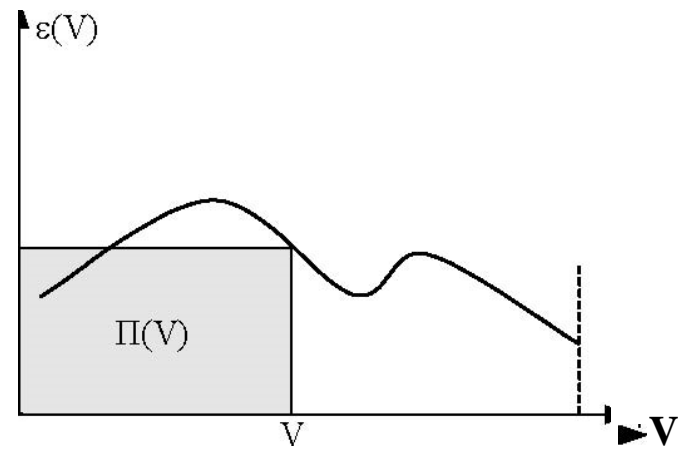

Рисунок 3 - Функция прибыли 
Из этого факта следует полезное свойство: если для двух точек $(V 1, \mathcal{E}(V 1)) u(V 2$, $\varepsilon(V 2))$ имеет место $V 2 \geq V 1$ u $\varepsilon(V 2) \geq \varepsilon(V 1)$, то, очевидно, решение $(V 2, \varepsilon(V 2))$ лучше, чем решение $(V l, \varepsilon(V 1))$. Это свойство позволяет перейти от зависимости $\varepsilon(V)$ к зависимости $\mathcal{E}^{*}(V)$, которая является убывающей функцией $V$. Способ построения зависимости $\mathcal{E}^{*}(V)$ ясен из рисунка 4 (зависимость $\mathcal{E}^{*}(V)$ показана толстой линией).

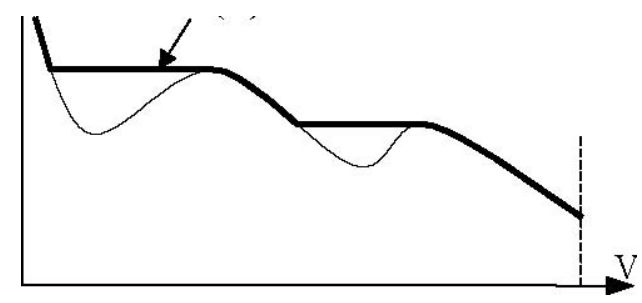

\section{Рисунок 4 - Построение функции прибыли}

Зависимость $\mathcal{E}(V)$ (а значит и $\mathcal{E}^{*}(V)$ ) является кусочно-постоянной, непрерывной слева функцией. Поэтому фактически следует сравнить конечное число вариантов. Приведем геометрическое правило, позволяющее сравнивать любые два варианта. Для этого запишем условие того, что вариант $\left(V_{l}, \mathcal{\varepsilon}_{l}\right)$ лучше варианта $\left(V_{2}, \varepsilon_{2}\right): V_{l} \varepsilon_{l}>V_{2} \varepsilon_{2}$.

Перепишем это условие в виде:

$$
\operatorname{tg} \alpha_{1}=\frac{\varepsilon_{1}}{V_{2}}>\frac{\varepsilon_{2}}{V_{1}}=\operatorname{tg} \alpha_{2}
$$

Геометрический смысл условия (5) ясен из Рисунок 5.

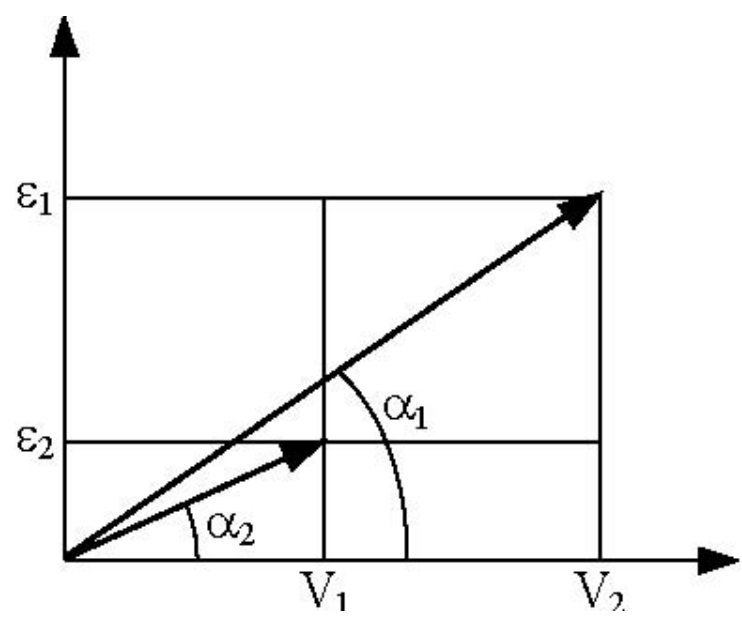

\section{Рисунок 5 - Геометрический смысл оптимальности}

Действительно, $\varepsilon_{1} / V_{2}$ равно тангенсу угла $\alpha_{1}$, а $\varepsilon_{2} / V_{1}$ равно тангенсу угла $\alpha_{2}$. Следовательно, вариант $\left(V_{1}, \varepsilon_{1}\right)$ лучше варианта $\left(V_{2}, \varepsilon_{2}\right)$, если угол $\alpha_{1}$ больше угла $\alpha_{2}$. Это наглядное правило позволяет решать задачу попарно сравнивая варианты. 


\section{Пример формирования ценовых механизмов}

Данные о предлагаемых потребителями ценах и величинах заказов приведены в таблице 1 , а данные об изменении оптовых цен производителя в зависимости от объема закупок центром - в таблице 2.

\section{Таблица 1 - Цены и спрос потребителей}

\begin{tabular}{|l|l|l|l|l|l|}
\hline $\mathrm{N}$ & 1 & 2 & 3 & 4 & 5 \\
\hline $\mathrm{vi}$ & 3 & 4 & 2 & 4 & 2 \\
\hline $\mathrm{ci}$ & 2 & 3 & 4 & 5 & 7 \\
\hline
\end{tabular}

Таблица 2 - Данные об изменении оптовых цен производителя

\begin{tabular}{|c|c|c|c|}
\hline $\mathrm{V}$ & $\mathrm{V}<5$ & $5 \leq \mathrm{V}<11$ & $\mathrm{~V} \geq 11$ \\
\hline $\mathrm{b}(\mathrm{V})$ & 4 & 2 & 1 \\
\hline
\end{tabular}

Сначала получаем зависимость $\mathrm{q}(\mathrm{V})$. Для этого при каждом значении $\mathrm{q}$ суммируем заказы всех потребителей, у которых предлагаемая ими цена сі больше или равна q. Так при q=4 величина $\mathrm{q}(\mathrm{V})$ равна сумме заказов третьего, четвертого и пятого потребителей, то есть, равна 8. В результате получаем таблицу 3.

\section{Таблица 3 - Суммарные заказы потребителей}

\begin{tabular}{|c|c|c|c|c|c|c|}
\hline $\mathrm{V}$ & $0<\mathrm{V} \leq 2$ & $2<\mathrm{V} \leq 6$ & $6<\mathrm{V} \leq 8$ & $8<\mathrm{V} \leq 12$ & $12<\mathrm{V} \leq 15$ & $15<\mathrm{V}$ \\
\hline $\mathrm{q}(\mathrm{V})$ & 7 & 5 & 4 & 3 & 2 & 0 \\
\hline
\end{tabular}

Вычитая из $q(V)$ (таблица 3) величину $b(V)$ (таблица 2) получаем таблицу 4 значений $\mathcal{E}(V)$ :

\section{Таблица 4 - Прибыль центра}

\begin{tabular}{|c|c|c|c|c|c|}
\hline $\mathrm{V}$ & 2 & 6 & 8 & 12 & 15 \\
\hline$\varepsilon(\mathrm{V})$ & 3 & 3 & 2 & 2 & 1 \\
\hline
\end{tabular}

В таблице 4 указаны значения $\mathcal{E}(V)$ только в точках $v_{i}$, то есть в точках, в которых происходит изменение величины $V$ (появляются новые потребители, согласные заключить договор с центром). Можно показать, что оптимальный объем заказа центра достигается только в этих точках. Действительно, если центру выгодно заключить договор с потребителем на частичное удовлетворение его потребностей в продукции, то центру еще более выгодно заключить договора с этим потребителем на полное обеспечение продукцией. 
Теперь, применяя описанные выше правила сравнения вариантов, сравниваем варианты последовательно, начиная с первого. Первый вариант хуже второго, поскольку $V_{1}<V_{2}$, а $\varepsilon_{1}=\varepsilon_{2}$. Второй вариант лучше третьего, так как $\varepsilon_{2} / V_{3}=3 / 8>\varepsilon_{3} /$ $V_{2}=1 / 3$. Второй вариант хуже четвертого, так как $\varepsilon_{2} / V_{4}=1 / 4<\varepsilon_{4} / V_{2}=1 / 3$. Наконец, четвертый вариант лучше пятого, так как $\varepsilon_{4} / V_{5}=2 / 15>\varepsilon_{5} / V_{4}=1 / 12$.

Итак, оптимальным является вариант 4, в котором по централизованной схеме обеспечиваются первые четыре потребителя.

При этом объем продукции, заказываемой центром, составляет 12 единиц, оптовая цена производителя равна 1 , а цена продукции центра равна 3. Прибыль центра составляет (3-1)·12 = 24 единицы.

\section{Заключение}

Рассмотренные в работе модели и механизмы управления материальнотехническим снабжением могут составить основу для создания систем поддержки принятия решений в этой области. Безусловно, многие задачи требуют дальнейших исследований. Особенно это относится к экспериментальной проверке различных механизмов деловых игр.

\section{Список информационных источников}

[1] Сатышев С.Н., Подпорин Д.И., Шарков А.А., Мазуренко С.В. Автоматизация процессов планирования и управления поставками продукции. //Аналитикоимитационное моделирование и ситуационное управление в промышленности, строительстве и образовании (ч.1): сб. науч. тр. МАДИ(ГТУ) / МАДИ(ГТУ). -М., 2008. -C.42-51.

[2] Шарков А.А. Имитационное моделирование процессов управления запасами предприятий технического обслуживания. // Вестник / МАДИ(ГТУ).-М., 2009. № 1(16). - C.102-104.

[3] Остроух А.В., Синха Бабу Раджа Исследование информационных систем управления взаимоотношениями с поставщиками // Автоматизация и управление в технических системах. - 2013. - № 4.1; DOI: 10.12731/2306-1561-2013-4-10; URL: auts.esrae.ru/6-124 (дата обращения: 22.11.2013).

[4] Остроух А.В. Информационные технологии в научной и производственной деятельности / [ред. А.В. Остроух] - М: ООО "Техполиграфцентр", 2011. - 240 с. ISBN 978-5-94385-056-1.

[5] Куфтинова Н.Г. Процессно-ориентированный подход к автоматизации планирования и управления транспортировкой продукции предприятий промышленности / А.В. Остроух, Н.Г. Куфтинова // Вестник МАДИ - 2010. - Вып. 4(23). - C. 62-66.

[6] Остроух А.В. Исследование начального периода моделирования на точность среднеинтегральной оценки имитационных моделей / А.В. Остроух, А.А. Солнцев, Н.В. Солдатов, К.А. Новицкий, П.С. Якунин // Вестник МАДИ - 2010. Вып. 2(21). - С. 61-65. 
[7] Остроух А.В. Математическая модель связей в системе диагностики электрооборудования автомобилей / А.В. Остроух, А.А. Солнцев, О.Ф. Калухов, Г.Г. Ягудаев // Вестник МАДИ - 2010. - Вып. 2(21). - С. 66-70.

[8] Николаев А.Б., Приходько М.В., Сатышев С.Н., Солнцев А.А., Якунин П.С. Имитационные модели оценки качества транспортного обслуживания. //Автотранспортное предприятие. -2013. - № 2. - С. 52-54.

[9] Николаев А.Б. Информационные технологии в менеджменте и транспортной логистике: учебное пособие / А.Б. Николаев, А.В. Остроух. - Saint-Louis, MO, USA: Publishing House Science and Innovation Center, 2013. - 254 c. - ISBN 978-0615-67110-9.

[10] Солнцев А.А., Ивахненко А.А. Формальное описание процессов движения комплектующих на основе управляемых сетей // Автоматизация и управление в технических системах. - 2013. - № 1(3); URL: auts.esrae.ru/3-69 (дата обращения: 19.11.2013). 